\title{
9 A USER INTERFACE FOR EMERGENT SEMANTICS IN IMAGE DATABASES
}

Simone Santini†, Amarnath Gupta ${ }^{\ddagger}$, and Ramesh Jain ${ }^{\dagger}$

†Visual Computing Laboratory, University of California, San Diego. \{ssantini,jain\}@cs.ucsd.edu

‡San Diego Supercomputer Center*

gupta@sdsc.edu

\begin{abstract}
In this paper, we discuss image semantics and the repercussions that its correct definition have on the design of image databases. We start by rejecting the simplistic notion that the meaning of an image is a function of the objects that the image contains, and show that meaning can only be defined in the context of a query, and can only be revealed in the context of the whole database.

With our definition, meaning is no longer a characteristic of the image that is extracted and compared in the querying process. Meaning is a product of the query process. In particular, meaning is emergent from the interaction of the user with the database. This state of affairs makes the interface one of the most crucial components in the database, since it is through the interaction that takes pace in the interface that the meaning of images is extracted. We propose a new model for interfaces that takes into account the necessity for the user to explore the database rather than passively asking questions and waiting for answers.
\end{abstract}

\subsection{INTRODUCTION}

In this paper we propose a new theory of image semantics, and study some of the consequences of the new definition on image databases. Most current image databases follow a model derived from traditional databases according to which

*This work was partially supported by NSF under grant NSF IRI-9610518 
the meaning of a record is a compositional function of the syntactic structure of the record and of the meaning of its elementary constituents. We show that this definition and its obvious extensions are inadequate to capture the real meaning of an image. Even if we could do perfect object recognition (which we can't), this would still not be enough to assign to images a semantics that satisfies the user of the database. The reason for this state of affair is that images are designed to convey a certain message, and the message is concealed in the whole organization of the image, and is not possible to divide it syntactically into smaller parts.

We propose that the meaning of an image is characterized by the following properties:

- It is contextual. The meaning of an image depends on the particular conditions under which the query is made, and the particular user that is querying the database.

- It is differential. The meaning of an image is apparent if the image is placed in the context of other similar images.

- It is grounded in action. The database can establish the meaning of an image based on the actions of the user when the image is presented. In a database situation, the only action allowed to the user is asking a query. Therefore, the meaning of an image will be revealed to the database by interpreting the sequence of queries posed by the user.

These ideas lead to the design of a different type of image database. In our system the semantics is not an intrinsic property of the images, but an emergent property of the interaction between the user and the database. The interface between man and machine assumes a preponderant role in this new organization.

This paper is organized as follows. In Section 9.2, we present an analysis of the meaning of records in traditional databases and show that this analysis cannot be extended to the meaning of images. Moreover, we present an alternative analysis of the meaning of an image and show how it depends on the actions of the user and on the relation between an image and the rest of the database. In Section 9.3 we present a formalization of the meaning of an image, and show how the meaning is altered by a certain class of operations on the feature space. In Section 9.4 we introduce informally our database interface based on a direct manipulation model. Our interface is directly related to our definition of meaning. In Section 9.5, we formalize the interface introduced in Section 9.4, and define it as a number of operators on three image spaces. In Section 9.6, we present an implementation of this interface in our system El Niño, and present some examples of extraction of meaning from a collection of images. Conclusions and future directions of research are presented in Section 9.7. 


\subsection{MEANING}

In most databases, the meaning of a record is a simple function of the syntactic structure of the record and of the meanings of its components. In other words, the meaning of a record is compositional. We have already pointed out in the previous section that, according to our point of view, the meaning of a stimulus in a given situation must be related to the set of possible actions of an actor in that situation. In a database situation, the only possible actions are asking queries and answering them. Then, if $\mathcal{Q}$ is the set of all possible queries, the meaning of a record, or a fragment of a record $R$, can be defined as a function

$$
[R]: Q \rightarrow\{\text { yes, no }\}
$$

such that $[R](q)=\{$ yes $\}$ if the record $r$ satisfies the query $q$. Compositionality implies that, if a record is produced by a rule like

$$
j: R \rightarrow \alpha_{1} R_{1} \alpha_{2} \cdots \alpha_{n} R_{n} \alpha_{n+1}
$$

where $\alpha_{i}$ are terminal symbols of the record language, and $R_{i}$ are non terminal symbols, and $j$ is the label of the production rule, then the meaning of $R$ is:

$$
[R]=f_{j}\left(\left[R_{1}\right],\left[R_{2}\right], \ldots,\left[R_{n}\right]\right) .
$$

The meaning of the whole record depends on the production rule and on the meaning of the non terminals on the right side of the production, but not on the syntactic structure of the non terminals.

This property makes the analysis of the meaning of records in traditional databases but, unfortunately, it does not hold for images. As [2] puts it:

The most naive way of formulating the problem is: are there iconic sentences and phonemes? Such a formulation undoubtedly stems from a sort of verbocentric dogmatism, but in it ingenuousness it conceals a serious problem.

The problem is indeed important and the answer to the question, as Eco points out, is "no." It is true that we can find certain semantic units in images in the form of objects, but there are two factors that prevent us from equating images and language sentences: objects are not further decomposable using linguistic means, and they do not fully represent the meaning of images.

In language we find the presence of verbal rules and discrete units at all levels. A tree defines the deep structure of a sentence (at least if we follow Chomsky's theory); a grammar transforms the deep structure into a series of words; phonetic rules govern the articulation of phonemes into words. On the iconic level, however, we face a much more complicated situation. We should regard images as "weak" codes [2], as opposed to the "strong" code represented by language. Although some images contain syntactical units in the form of objects, underneath this level it is no longer possible to continue the subdivision.

In addition to this, objects are not sufficient to encode the meaning of an image. Consider the two images of Fig. 9.1, both of which contain essentially 

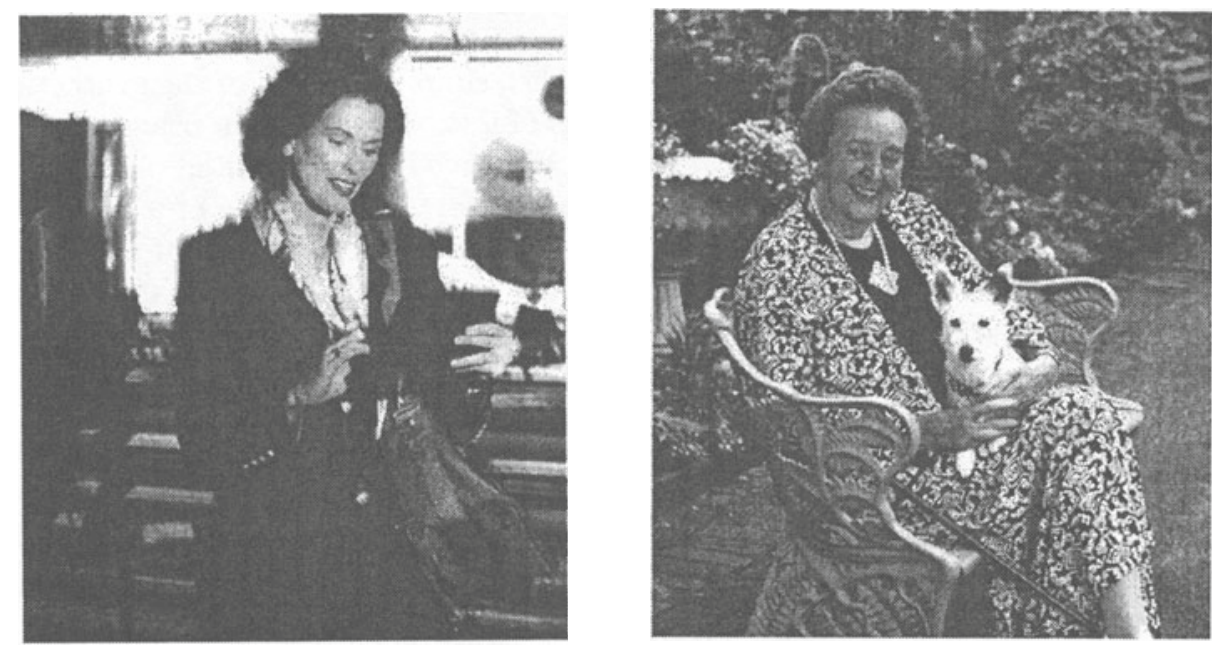

Figure 9.1: Two images responding to the query "image of a woman" with very different semantics.

a single object that can be characterized as "a woman." According to the simplistic interpretation, these two images have the same semantics. However, if looked in the context of our culture and society, they are very different. One of these two images conveys a sense of activity: it is obvious that the woman in the first image has very little time to waste, and is attending some important and urgent business. The second image conveys a certain bucolic tranquillity. We can imagine that the woman in the second image can make an excellent tea

Semantic level beyond the objects are used very often in evocative scenarios, like art and advertising [1]. There is, for instance, a fairly complex theory of the semantics associated with color [5], and with certain representational conventions [3].

The full meaning of an image depends not only on the image data, but on a complex of cultural and social conventions in use at the time and location of the query, as well as on other contingencies of the context in which the user interacts with the database. This leads us to reject the somewhat Aristotelean view that the meaning of an image is an immanent property of the image data. Rather, the meaning arises from a process of interpretation and is the result of the image data and the perceptual processes of the observer. The process of querying the database should not be seen as an operation during which images are filtered based on the illusory pre-existing meaning but, rather, as a process in which meaning is created through the interaction of the user and the images.

This example also reveals another aspect of the image-sign: its conventionality. Although the contents of an image are not arbitrary in the way in which the Saussurean sign is [12], they are still produced through a convention: they are cultural manifestations [2]. 


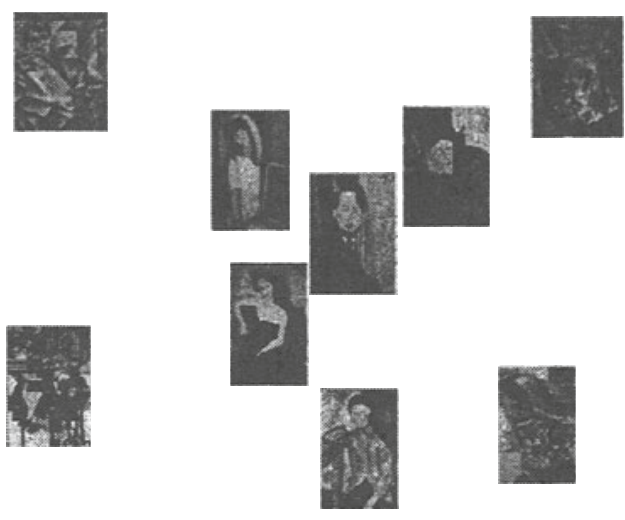

Figure 9.2: A Modigliani portrait placed in a context that suggests "Painting."
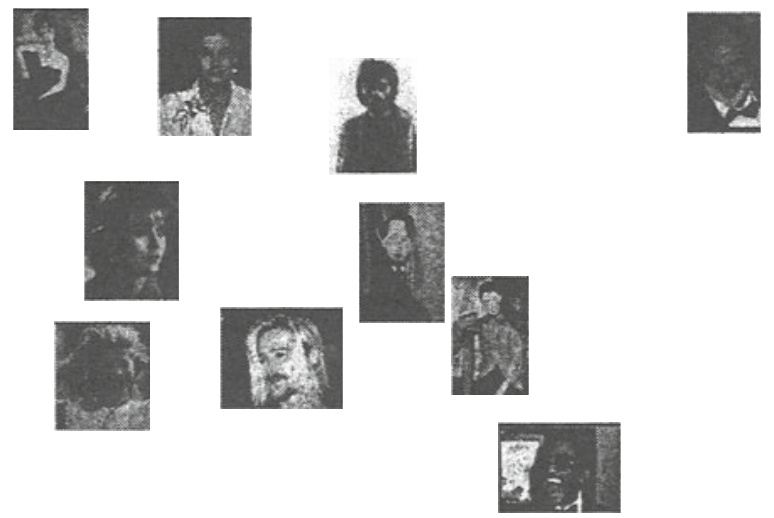

Figure 9.3: A Modigliani portrait placed in a context that suggests "Face."

Consider the images of Fig. 9.2. The image at the center is a Modigliani portrait and, placed in the context of other 20th century paintings (some of which are portraits and some of not), suggests the notion of "painting." If we take the same image and place it in the context of Fig. 9.3, the context suggests the meaning "Face." 
We are now caught in the middle between two tractable but unattainable opposites. On one hand, images are not linguistic signs that is, their meaning can't be described by a grammar and resolved using standard parsing techniques.

On the other hand, semantics is not a simple function of image contents at all. In particular, we can't hope to find intrinsically determined units of meaning in the image data. The meaning of the image data can only result from the interaction with the user. The user will provide the cultural background in which meaning can be grounded. We call this concept emergent semantics. The semantics of an image is not a property of the image per se, but something that emerges from the interaction of the user with the database. This concept has important consequences for our query model and, ultimately, for the architecture of image databases.

In traditional database, the assumption that the meaning is a property of the image leads us to the the query process as a filter. We take all the records, filter out those that don't have the required meaning, and show the others. If meaning is the result of user interaction, this approach is no longer possible. Rather, querying should be seen as a process of exploration and reorganization of the database.

In this approach, the interface between the user and the database is of primary importance. The interface is no longer the place where questions are asked and answers are obtained, but it is the tool for active manipulation of the database as a whole. We present our approach to interfacing, called direct manipulation in Sect. 9.4. Before that, in the next section, we will briefly formalize the definition of meaning that we have outlined in this section.

\subsection{MEANING. A FORMAL MODEL}

As discussed in the previous section, the meaning of an image is a set of relations between that image and the other images in the database. These relations are expressed as dissimilarity, or distance between two images. The distance function in the image space, on the other hand, is determined by the specific query that the user is asking. As we will see more in detail in the next section, a query $q$ endows the feature space in which images are described with a metric $g(q)=\left\{g_{i j}(q)\right\}$ which, in turn, can be described by a finite number of parameters $\xi^{\mu}, \mu=1, \ldots, m$. The metric $g$ determines the distance and the relation between images.

Let $\mathcal{I}$ be the set of images in the database, and $\mathcal{Q}$ the set of queries. Image $I \in \mathcal{I}$ is described by a feature vector $x_{I}$. Given the metric $g(q)$, the distance between two images is a function

$$
f\left(x_{I}, x_{J} ; g(q)\right)=\int_{x_{I}}^{x_{J}}\left(\sum_{i j} g_{i j}(q) \dot{x}^{i} \dot{x}^{j}\right)^{\frac{1}{2}} d t
$$


where the integral is computed along a curve $x(t)$ which is a geodesic between $x_{I}$ and $x_{J}$ [7]. The semantic difference between images $I$ and $J$ is a function

$$
m_{I, J}: \mathcal{Q} \rightarrow \mathrm{R}^{+}: q \mapsto \frac{f\left(x_{I}, x_{J} ; \xi^{\mu}(q)\right)}{f\left(0, x_{I} ; \xi^{\mu}(q)\right)}
$$

We can generalize our definition and make it independent on the particular contents of the database. We can imagine that every point in $\mathbf{R}^{n}$ contains an image, and define the difference in meaning between points $x$ and $y$ as

$$
m_{x y}: \mathcal{Q} \rightarrow \mathbf{R}^{+}: q \mapsto \frac{f\left(x, x ; \xi^{\mu}(q)\right)}{f\left(0, x ; \xi^{\mu}(q)\right)}
$$

The meaning of point $x \in \mathrm{R}^{n}$ is a function $m_{I} \in L^{2}\left(\mathrm{R}^{n} \times \mathcal{Q}, \mathbf{R}^{+}\right)$defined as:

$$
m_{x}(y, q)=m_{x y}(q)
$$

and the meaning assignment operator is

$$
\mu: \mathbf{R}^{n} \rightarrow L^{2}\left(\mathbf{R}^{n} \times \mathcal{Q}, \mathbf{R}^{+}\right): x \mapsto m_{x}
$$

With these definitions, the meaning of an image is no longer a "thing" contained in the image or a function of the image data alone. The meaning depends on the whole distribution of images in the database (via the image $y$ in (9.7) and on the metric induced by the current query (via the parameters $\xi^{\mu}$ in (9.6)).

The meaning of an image depends on the query that is being asked. Once a query has been specified, the meaning can be grounded to that query by the operator $\gamma: \mathcal{Q} \rightarrow L^{2}\left(L^{2}\left(\mathbf{R}^{n} \times \mathcal{Q}, \mathbf{R}^{+}\right), L^{2}\left(\mathbf{R}^{n}, \mathbf{R}\right)\right)$ defined as:

$$
\gamma(q)\left(m_{x}\right)=f\left(x, \cdot ; \xi^{\mu}\right)
$$

The operator $\Gamma$ is the grounded meaning assignment. Given a query $q$, the meaning of an image $x$ with respect to $q$ is

$$
\Gamma_{q, x}=\Gamma(q, x)=f\left(x, ; \xi^{\mu}(q)\right)
$$

We also call this function the configuration of the images with respect to the point $x$ and query $q$.

This definition of meaning is an important step to understand the inadequacy of the traditional query model when applied to image database. Most of the problems come from the different definitions that apply in the two cases. The theory of meaning presented here forms a basis for the concept of emergent semantics that will lead to the definition of direct manipulation interfaces in the following section.

Semantics is emergent in the sense that it can only be defined with respect to the distribution of images induced by a particular query. Therefore, the process of query making is not just a filter that selects some of the images based on a pre-existing meaning, but a "conversational" activity during which the meaning of the images is created. The following section will introduce informally our interface and, hopefully, make this concept clearer. 


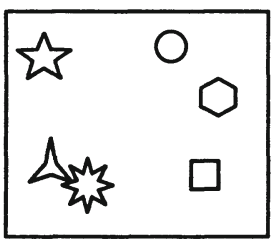

(A)

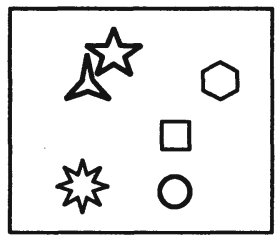

(C)

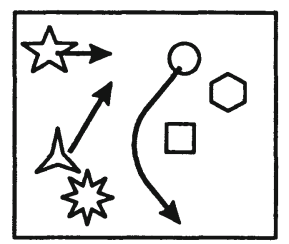

(B)

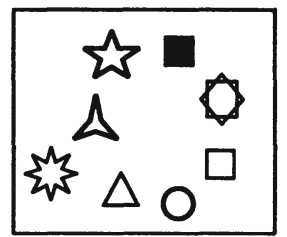

(D)

Figure 9.4: Schematic description of an interaction using a direct manipulation interface.

\subsection{EMERGENT SEMANTICS: AN INTERFACE PROPOSAL}

From the considerations of the previous two sections it appears that the user plays an essential role in determining the meaning of an image in a specific situation and the similarity between two images. In the example of the two women above, the user should provide the context to decide whether the "woman" aspect of the images is prevailing (in which case the two images should be considered fairly similar) or the "business" aspect should prevail (in which case the two images would not be considered similar.)

Based on this principle, we replaced the query-answer model of interaction with direct manipulation. In our model, the database gives information about the status of the whole database, rather than just about a few images that satisfy the query. Whenever possible, the user manipulates the image space directly by moving images around, rather than manipulating weights or some other quantity related to the current similarity measure. The manipulation of images in the display causes the creation of a similarity measure that satisfies the relations imposed by the user. Rather than the user trying to understand the properties of the similarity measures used by the database, the database should use the user categorization to develop new similarity measures.

An user interaction using a direct manipulation interface is shown schematically in Fig. 9.4. In Fig. 9.4.A the database proposes a certain distribution of images (represented schematically as colored rectangles) to the user. The distribution of the images reflects the current similarity interpretation of the database. For instance, the green image is considered very similar to the orange one, and the brown to the purple. In Fig. 9.4.B the user moves some images around to reflect his own interpretation of the relevant similarities. The result 


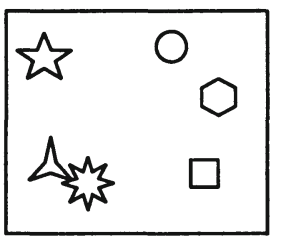

(A)

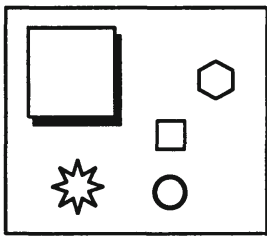

(C)

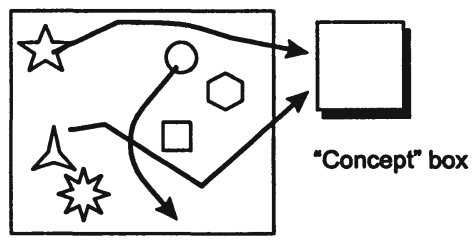

(B)

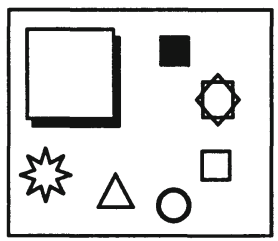

(D)

Figure 9.5: Interaction involving the creation of concepts.

is shown in Fig. 9.4.C. According to the user, the red and green images are quite similar to each other, and the brown image is quite different from them.

As a result of the user assessment, the database will create a new similarity measure, and re-order the images, yielding the configuration of Fig. 9.4.D. The red and the green images are in this case considered quite similar (although the green image has been moved from its intended position), and the brown quite different. Note that the result is not a simple rearrangement of the images in the interface. For practical reasons, an interface can't present more than a small fraction of the images in the database. Typically, we display the 100-300 images most relevant to the query. The reorganization consequent the user interaction involves the whole database. Some images will disappear from the display (the purple image in Fig. 9.4.A), and some will appear (the yellow, gray, and cyan images in Fig. 9.4.D).

A slightly different operation on the same interface is the definition of visual concepts. In the context of direct manipulation, the term concept has a more restricted scope than in the common usage. A visual concept is simply a set of images that, for the purpose of the current application, can be considered as equivalent or almost equivalent. Images forming a visual concept can be dragged into a "concept box" and, if necessary, associated with some text (the text can be used to retrieve the concept and the images similar to it). The visual concept can be then transformed into an icon and placed on the screen like every other image.

Fig. 9.5 is an example of interaction involving the creation of visual concepts. Fig. 9.5.A contains the answer of the database to a user query. The user considers the red and green images as two instances of a well defined linguistic concept. The user opens a concept box and drags the images inside the box. The boxes then used as an icon to replace the images in the display space. 
From the point of view of the interface, a concept is a group of images that occupy the same position in the display space. In addition, it is possible to attach meta-data information to a concept. For instance, if a user is exploring an art database, he can create a concept called "medieval crucifixion." The words "medieval" and "crucifixion" can be used to replace the actual images in a query. This mechanism gives a way of integrating visual and non visual queries. If an user looks for medieval paintings representing the crucifixion, she can simply type in the words. The corresponding visual concept will be retrieved from memory, placed in the visual display, and used as a visual query (see below for a more detailed introduction to the use of visual concepts for the integration of meta-data).

It is interesting to note that the distinction between the role of the user and the role of the database is blurred in this model. In very general terms, the role of the database is to focus the attention of the user on certain relations that, given the current interpretation of meaning in the database, are deemed relevant. The database does this by displaying a subset of relevant images and their relations in the similarity criterion that is used to define the meaning.

The role of the user is exactly the same. By displacing images in the interface plane, the user focuses the attention on the database on certain relation between images that, given the user interpretation of the meaning of the displayed images, are relevant. Both systems, the database and the user, will adjust their similarity measure based on the response of the other system. The fact that we expect more flexibility from the database rather than from the user (i.e. the database should adapt its similarity measure, while the user has a relatively stable idea of what he/she wants) makes the difference between the two a matter of degree rather than a categorical distinction.

Direct manipulation requires a different and more sophisticated organization of the database:

1. Manipulation of a contextual representation a formal definition of one or more display spaces, in which manipulation takes place. The display space should present images in a usable format, while retaining as much as possible the distance induced by the query.

2. The database must accommodate arbitrary (or almost arbitrary) similarity measures, and must automatically determine the similarity measure based on the user interface.

In the following section we describe in greater details the principles behind the design of direct manipulation interfaces. The focus of this paper is in interfaces, so we will not consider point 2 above in any detail. We will just make the necessary assumption about the similarity measures used by the system without explaining how these measures are implemented. The reader should refer to $[10,11]$ for more details. 


\subsection{DIRECT MANIPULATION INTERFACE}

The direct manipulation interface is composed of three spaces and a number of operators [4]. The operators can be transformations of a space onto itself or transformations from one space to another. The three spaces on which the interface is based are:

- The Feature space $\mathcal{F}$. This is the space of the coefficients of a suitable representation of the image. The feature space is a topological space, but not a metric one. There is in general no way to assign a "distance" to a pair of feature vectors.

- The Query space $\mathcal{Q}$. When the feature space is endowed with a metric, the result is the query space. The metric of the query space is derived from the user query, so that the distance from the origin of the space to any image defines the "dissimilarity" of that image from the current query.

- The Display space $\mathcal{D}$ is a low dimensional space (0 to 3 dimensions) which is displayed to the user and with which the user interacts. The distribution of images in the display space is derived from that of the query space. We will mainly deal with two-dimensional display spaces (as implemented in a window on a computer screen.) For the sake of convenience, we also assume that every image in the visualization space has attached a number of labels $\lambda_{i}$ drawn from a finite set. Examples of labels are the visual concepts to which an image belongs. The conventional label $\alpha$ is assigned to those images that have been placed in their position by the user.

The feature space is a relatively fixed entity, and is a property of the database. The query space, on the other hand, is created anew with a different metric for every new query. Note that the feature space is not completely immutable. Some of the operators presented in the following operate on the feature space. This is usually done for reasons of convenience (some queries may be faster on some particular transformation of the feature space), a concept not dissimilar from the formation of "stored views" in databases.

\subsubsection{Oper tors in the Feature Space}

A feature is an attribute obtained applying some image analysis algorithm to the image data. Features are often collected in a feature vector, an immersed in a suitable vector space, although this is not always the case. In El Niño an image is represented by a set of coefficients, each belonging to a relatively low dimensional space [10].

We make a distinction between the raw, unprocessed vector space and spaces that are adapted from it for reasons of convenience. This distinction is not fundamental (all feature spaces are the result of some processing) but it will be useful to describe the operators. The raw feature space is the set of complete feature vectors, as they come out of the image analysis algorithm. In many 
cases, we need to adjust these vectors for the convenience of the database operations. A very common example is dimensionality reduction [8]. In this case, we will say that we obtain a view in the feature space. The operators that operate on the feature space are used for this purpose. The most common are:

Projection.. The feature vector is projected on a low dimensional subspace of the raw feature space, obtaining a low dimensional view. Operators like Singular Value Decomposition, projection of Zernike moments, and of statistical moments belong to this class.

Quantization.. These operators are used in non-vector feature spaces like the set of coefficients used in El Niño. In this case, we reduce the dimensionality of the feature space by representing an image with a limited number of coefficients (e.g. 50 or 100). This is done by vector quantization of the image coefficients.

Apply Function.. Applies the function $F$ to all the elements of a set of numbers to create another set of numbers of the same dimension. Filtering operations applied to color histograms belong to this class.

These operators "prepare" the feature space for the database operations. They are not properly part of the interaction that goes on in the interface, since they are applied off-line before the user starts interacting. We have mentioned them anyway for the sake of completeness.

\subsubsection{The Query Space}

The feature space, endowed with a similarity measure derived from a query, becomes the query space. The "score" of an image is determined by its distance from the origin. The determination of the geometry of the query space is in general quite complicated, and is beyond the scope of this paper. We will just assume that every image is represented as a set of $n$ number (which may or may not identify a vector in an $n$-dimensional vector space, as discussed in the previous section) and that the query space is endowed with a distance function that depends on $m$ parameters.

The feature sets corresponding to images $x$ and $y$ are represented by $x^{i}$ and $y^{i}, i=1, \ldots n$, and the parameters by $\xi^{\mu}, \mu=1, \ldots, m$. Also, to indicate a particular image in the database we will use either different Latin letters, as in $x^{i}, y^{i}$ or an uppercase Latin index. So $x_{I}$ is the $I$-th image in the database $(1 \leq I \leq N)$, and $x_{I}^{j}$ is the corresponding feature vector. Since this notation can be quite confusing, we will try to avoid it whenever possible, and use $x^{i}, y^{i}$ instead.

The parameters $\xi^{\mu}$ are a representation of the query, and are the values that determine the distance function.

Given the parameters $\xi^{\mu}$, the distance function in the query space can be written as

$$
f: \mathbf{R}^{n} \times \mathbf{R}^{n} \times \mathbf{R}^{m} \rightarrow \mathbf{R}^{+}:\left(x^{i}, y^{i}, \xi^{\mu}\right) \mapsto f\left(x^{i}, y^{i} ; \xi^{\mu}\right)
$$


with $f \in L^{2}\left(\mathbf{R}^{n} \times \mathbf{R}^{n} \times \mathbf{R}^{m}, \mathbf{R}^{+}\right)$. Depending on the situation, we will write $f_{\xi}\left(x^{i}, y^{i}\right)$ in lieu of $f\left(x^{i}, y^{i} ; \xi^{\mu}\right)$.

As stated in the previous section, the feature space per se is topological but not metric. Rather, its intrinsic properties are characterized by the functional

$$
L: \mathbf{R}^{m} \rightarrow L^{2}\left(\mathbf{R}^{n} \times \mathbf{R}^{n}, \mathbf{R}^{+}\right)
$$

which associates to each query $\xi^{\mu}$ a distance function:

$$
L\left(\xi^{\mu}\right)=f\left(\cdot, \cdot ; \xi^{\mu}\right) .
$$

A query $q$, characterized by a vector of parameters $\xi^{\mu}$, can also be seen as an operator $q$ which transforms the feature space into the query space. If $L$ is the characteristic functional of the feature space, then $q L=L(\xi)$ is the metric of the query space.

Once the feature space $\mathcal{F}$ space has been transformed into the metric query space $Q$, other operations are possible [4], like:

Distance.. Given a feature set $x^{i}$, return its distance from the query:

$$
D\left(x^{i}\right)=f\left(0, x^{i} ; \xi^{\mu}\right)
$$

Select by Distance.. Return all feature sets that are closer to the query than a given distance:

$$
S(d)=\left\{x^{i}: D\left(x^{i}\right) \leq d\right\}
$$

$k$-Nearest Neighbors.. Return the $k$ images closest to the query

$$
N(k)=\left\{x^{i}:\left|\left\{y^{i}: D\left(y^{i}\right)<D\left(x^{i}\right)\right\}\right|<k\right\}
$$

It is necessary to stress again that these operations are not defined in the feature space $\mathcal{F}$ since that space is not endowed with a metric. Only when a query is defined does a metric exist.

\subsubsection{The Display Space}

The display operator $\phi$ projects image $x^{i}$ on the screen position $X^{\Psi}, \Psi=1,2$ in such a way that

$$
d\left(X^{\Psi}, Y^{\Psi}\right) \approx f\left(x^{i}, y^{i} ; \xi^{\mu}\right)
$$

In El Niño, we use a simple elastic model to determine the position of images in the display space. First, we query the database so as to determine the $P$ images closer to the query. The display space will be concerned only with these images. In general, we use $100 \leq P \leq 300$. A few hundred images are in general sufficient to give the user a fair idea of the distribution of images, and don't clutter the display with irrelevant information. Let $f\left(x_{I}^{k}, x_{J}^{k} ; \xi^{\mu}\right)$, $I, J \leq P$ be the distance between the I-th and the J-th image in the database, with $0 \leq f\left(x_{I}^{k}, x_{J}^{k} ; \xi^{\mu}\right) \leq 1$. Also, let $X_{I}^{\Psi}$ be the coordinates of the $I$-th image 
in the display space, and $d\left(X_{I}^{\Psi}, Y_{I}^{\Psi}\right)$ the Euclidean distance between images $I$ and $J$ in the display space. We imagine to attach a spring of length $d\left(X_{I}^{\Psi}, Y_{I}^{\Psi}\right)$ between images $I$ and $J$. In a given configuration $\left\{X_{I}^{\Psi}, i=1, \ldots, P\right\}$ the energy of the system is proportional to:

$$
E=\sum_{i, j=1}^{Q}\left(d\left(X_{I}^{\Psi}, Y_{I}^{\Psi}\right)-f\left(x_{I}^{k}, x_{J}^{k} ; \xi^{\mu}\right)\right)^{2}
$$

We can use standard optimization techniques to solve this optimization problem and find the optimal configuration of the display space. More sophisticated methods can be used depending on the situation. The result is an operator that we write:

$$
\Psi\left(x_{I}^{k} ; f_{\xi}\right)=\left(X_{I}^{\Psi}, \emptyset\right) .
$$

The parameter $f_{\xi}$ reminds us that the projection that we see on the screen depends on the distribution of images in the query space which, in turn, depends on the query parameters $\xi^{\mu}$. The notation $\left(X_{I}^{\Psi}, \emptyset\right)$ means that the image $x_{I}$ is placed at the coordinates $x_{I}^{\Psi}$ in the display space, and that there are no labels attached to it (that is, the image is not anchored at any particular location of the screen, and does not belong to any particular visual concept).

A configuration of the display space is obtained by applying the display operator to the whole query space:

$$
\phi(\mathcal{Q})=\phi\left(\mathcal{F} ; f_{\xi}\right)=\left\{\left(X_{I}^{\Psi}, \aleph_{I}\right)\right\}
$$

where $\aleph_{I}$ is the set of labels associated to image $I$. As we said before, it is impractical to display the whole database. More often, we display only a limited number $P$ of image. Formally, this can be done by applying the $P$ nearest neighbors operator to the space $\mathcal{Q}$ :

$$
\phi(N(P)(\mathcal{Q}))=\phi\left(N(P)\left(\mathcal{F} ; f_{\xi}\right)\right)=\left\{\left(X_{I}^{\Psi}, \aleph_{I}\right), i=1, \ldots, P\right\}
$$

where $\aleph_{I}$ is the set of labels associated to the $I$-th images. The display space $\mathcal{D}$ is the space of such configurations.

With these definitions, we can describe the operators that manipulate the display space.

The Place Operator. The place operator moves an image from one position of the display space to another, and attaches a label $\alpha$ to the images to "glue" it to its new position. The operator that places the $I$-th image in the display is $\zeta_{I}: \mathcal{Q} \rightarrow \mathcal{Q}$ with:

$$
\zeta_{I}\left\{\left(X_{J}^{\Psi}, \aleph_{J}\right)\right\}=\left(\left\{\left(X_{J}^{\Psi}, \aleph_{J}\right)\right\}-\left\{\left(X_{I}^{\Psi}, \aleph_{I}\right)\right\}\right) \cup\left\{\left(\tilde{X}_{I}^{\Psi}, \aleph_{I} \cap \alpha\right)\right\}
$$

where $\tilde{X}$ is the position given to the image by the user. 
Visual Concept Creation. A visual concept is is a set of images that, conceptually, occupy the same position in the display space and are characterized by a set of labels. Formally, we will include in the set of labels the keywords associated to the concept as well as the identifiers of the images that are included in the concept. So, if the concept contains images $I_{1}, \ldots, I_{k}$, the set of labels is

$$
\lambda=\left(W,\left\{I_{1}, \ldots, I_{k}\right\}\right)
$$

where $W$ is the set of keywords. We call $\Lambda$ the set of concept, and we will use the letter $\lambda$ to represent a concept.

The creation of a concept is an operator $\kappa: \mathcal{D} \rightarrow \Lambda$ defined as:

$$
\kappa\left\{\left(X_{J}^{\Psi}, \aleph_{J}\right)\right\}=W \cup\left\{I_{1}, \ldots, I_{k}\right\}=\lambda
$$

Visual Concept placement. The insertion of a concept in a position $Z^{\Psi}$ of the display space is defined as the action of the operator $\eta: \Lambda \times \mathbf{R}^{2} \times \mathcal{D} \rightarrow \mathcal{D}$ defined as:

$$
\eta\left(\Lambda, Z^{\Psi},\left\{\left(X_{J}^{\Psi}, \aleph_{J}\right)\right\}\right)=\left(\left\{\left(X_{J}^{\Psi}, \aleph_{J}\right)\right\}-\left\{\left(X_{I_{k}}^{\Psi}, \aleph_{J}\right)\right\}\right) \cup\left\{Z^{\Psi}, \alpha \cup \lambda\right\}
$$

Meta-data Queries. Visual concepts can be used to create visual queries based on semantic categories. Suppose a user enters a set of words $A$. It is possible to define the distance from the set $A$ to a visual concept $\lambda$ using normal information retrieval techniques [9]. Let $d(A, \lambda)$ be such a distance. Similarly, it is possible to determine the distance between two concepts $d\left(\lambda_{1}, \lambda_{2}\right)$. Then the textual query $A$ can be transformed in a configuration of the display space

$$
\left\{X_{I}^{\Psi}, \alpha \cup \lambda_{I}\right\}
$$

where

$$
\left[\sum_{\Psi}\left(X_{I}^{\Psi}\right)^{2}\right]^{\frac{1}{2}} \approx d\left(A, \lambda_{I}\right)
$$

and

$$
\left[\sum_{\Psi}\left(X_{I}^{\Psi}-X_{J}^{\Psi}\right)^{2}\right]^{\frac{1}{2}} \approx d\left(\lambda_{I}, \lambda_{J}\right)
$$

In other words, we can use the distance between the concepts and the query, as well as the distances between pairs of concepts, to place the corresponding images in the display space, thus transforming the textual query in a visual query.

\subsubsection{Query Creation}

When the user moves images around the interface, he or she imposes a certain number of constraints of the form $d\left(x^{I}, y^{I}\right)=d_{x y}$. Assume that the user takes a set $T$ of images and places them in certain positions of the interface, so that, 
for all pairs $(x, y) \in T \times T$, the value $d_{x y}$ is given. The query can then be determined by solving the system of equations:

$$
f\left(x^{i}, y^{i} ; \xi^{\mu}\right)=d_{x y} \quad x, y \in T
$$

in the unknown $\xi^{\mu}$.

Depending on the number of images that the user has placed (that is, the number of elements in $T$ ) the system (9.29) can be under-determined or overdetermined. Even if the system is under-determined, the particular form of the function $f$ might prevent us to find an exact solution. In these cases, it is useful to determine a least squares solution.

In any case, the creation of a query can be seen as an operator

$$
\chi: \mathcal{D} \rightarrow \mathbf{R}^{m}:\left\{\left(X_{I}^{\Psi}, \aleph_{I}\right)\right\} \mapsto \xi^{\mu} .
$$

In general, we require that a query depends only on the images that have been anchored by the user, so, if $C=\left\{\left(X_{I}^{\Psi}, \aleph_{I}\right)\right\}$ and $C^{\prime}=\left\{\left(X_{I}^{\Psi}, \aleph_{I}\right) \in C: \alpha \in \aleph_{I}\right\}$ we have $\chi(C)=\chi\left(C^{\prime}\right)$.

\subsection{THE INTERFACE AT WORK}

We have used these principles in the design of the interface for our database system El Niño. As we mentioned in the previous section, the interface that we described requires the support of a suitable engine and data model. In particular, the engine must be able to

- Understand the placement of images in the display space.

- Be able to create a similarity criterion "on the fly" based on the placement of samples in the display space.

The engine that we use in El Niño satisfies these requirements using a purely geometric approach. The feature space is generated with a multi-resolution decomposition of the image. Depending on the transformation group that generates the decomposition, the space can be embedded in different manifolds. If the transformation is generated by the two dimensional affine group, then the space has dimensions $x, y$, and scale, in addition to the three color dimensions $R, G, B$. In this case the feature space is diffeomorphic to $\mathrm{R}^{6}$.

In other applications, we generate the transform using the phase space of the Weyl-Heisenberg group [10], obtaining transformation kernels which are a generalization of the Gabor filters [6]. In this case, in addition to the six dimensions above we have the direction $\theta$ of the filters, and the feature space is diffeomorphic to $\mathrm{R}^{6} \times S^{1}$.

An image is represented as a set of coefficients in this six (or seven) dimensional space. The raw feature space of $\mathrm{El} \mathrm{Niño} \mathrm{is} \mathrm{the} \mathrm{space} \mathrm{of} \mathrm{such} \mathrm{sets} \mathrm{of}$ coefficients. Each image is represented by a set of about 30,000 coefficients. In order to reduce the memory occupation of each image and make the distance 

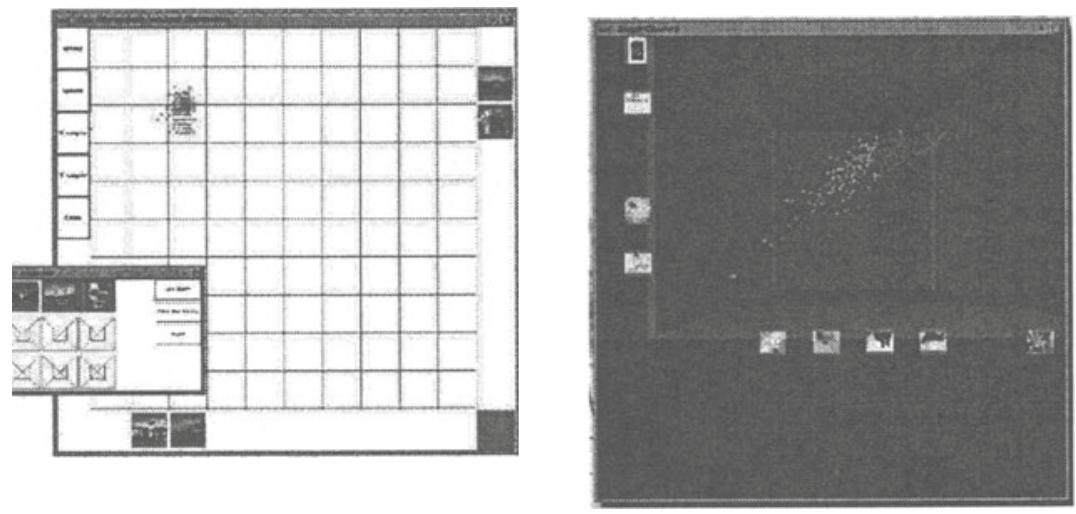

Figure 9.6: Two and three dimensional embodiments of the display space.

computations more efficient, we create a view in this space by a vector quantization operation that reduces an image to a number of coefficients between 50 and 100 (depending on the particular implementation of El Niño).

The query space is created endowing the view on the feature space with a metric. One of the characteristics of $\mathrm{El} \mathrm{Niño} \mathrm{is} \mathrm{that} \mathrm{the} \mathrm{metric} \mathrm{is} \mathrm{not} \mathrm{a} \mathrm{simple}$ Minkowski metric, but a more general Riemann metric. This fact allows us to create endless similarity criteria based on the query choices of the user. The description of the engine of El Niño goes beyond the scope of this paper. The interested reader can find a full description in [10].

The display space in El Niño can be visualized using a number of twodimensional displays, and some three dimensional displays. Fig. 9.6 shows a two dimensional display of El Niño. The user can zoom in and out, and see a sample of the images in the display along the two axes (at higher magnification the dots inside the display also are displayed as images). The small window on the left is a visual concept being formed. Other possible displays include a "checkerboard" display, in which images are displayed in a fixed grid a three dimensional display (Fig. 9.6.b).

To give an example of a typical interaction session with El Niño, consider a query in which we are looking for some old cars. At the beginning our ideas are quite fuzzy, and we set to explore the database. We have a few cars in our "labeled" subset of the database, and we start defining the concept of car as in Fig. 9.7. The result of a query using this concept is shown in Fig 9.8 This answer is not satisfactory, but it contains the seeds from which we can proceed towards more interesting areas of the image space. We select a few of the cars in the display and add them to the concept of car. We go through the stage of Fig. 9.9 until, at the end of our query, our concept of "car" has become that of Fig. 9.10. During this interaction, our idea of what would be an answer to the 


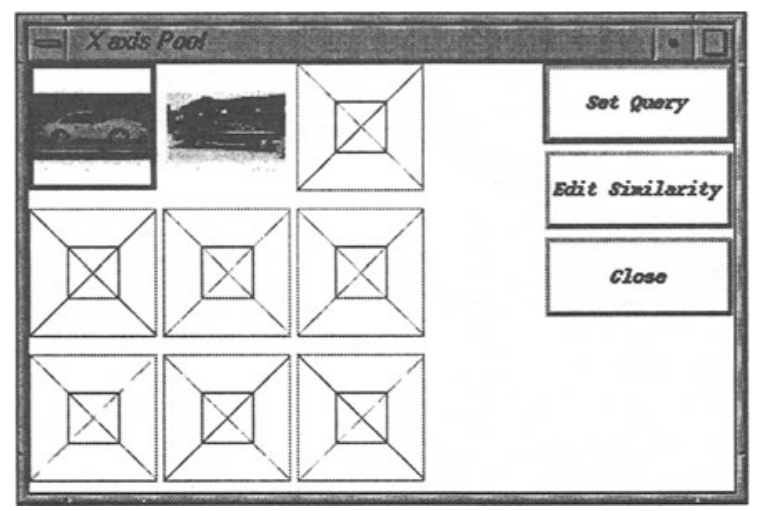

Figure 9.7: The initial concept of car that we use.

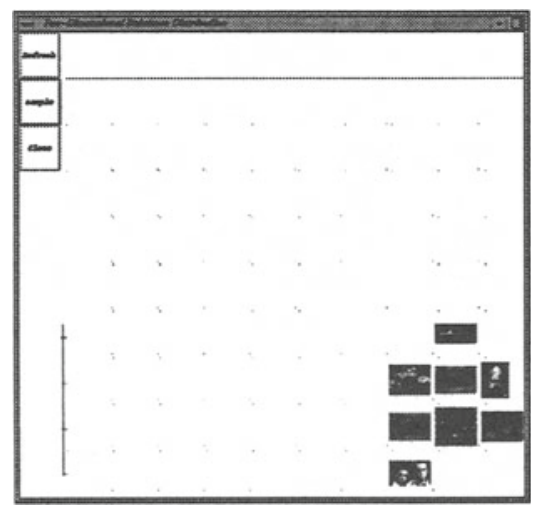

Figure 9.8: The result of a query with our first "car" concept. 


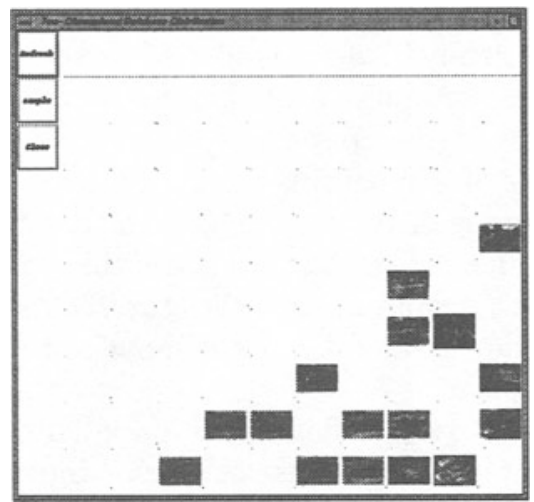

Figure 9.9: The result of a query with our second "car" concept.

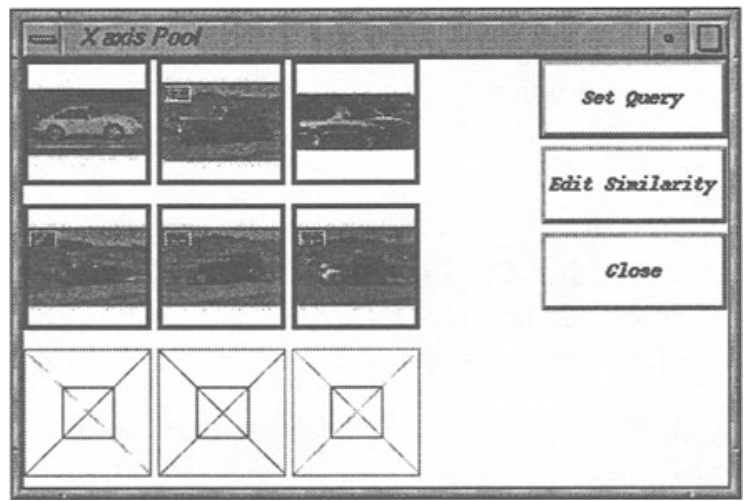

Figure 9.10: The final concept of car after the interaction. 
query changed continuously as we learned what the database had to offer, and redefined our goals based on what we saw.

\subsection{CONCLUSIONS}

In this paper we have defined a new model of interface for image databases. The motivation for the introduction of this model comes from an analysis of the semantics of images in the context of an image database. In traditional databases, the meaning of a record is a function from the set of queries to a set of truth values. The meaning of an image, on the other hand, is a function from the Cartesian product of the feature space times the set of queries to the positive real values. This definition embodies the observation that the meaning of an image can only be revealed by the comparison of an image with other images in the feature space.

These observations led us to define a new paradigm for database interfaces in which the role of the user is not just asking queries and receiving answers, but a more active exploration of the image space. The meaning of an image is emergent, in the sense that it is a product of the dual activities of the user and the database mediated by the interface.

We have proposed a model of interface for active exploration of image spaces. In our interface, the role of the database is to focus the attention of the user on certain relation that, given the current database interpretation of image meanings, are relevant. The role of the user is exactly the same: by moving images around, the user focuses the attention of the database on certain relations that, given the user interpretation of meaning, are deemed important.

Our future plans are to include different access modalities to the data into a single interface. Images accessed by keywords can be placed in the interface just like images accessed by visual features. Also, we plan on building more perceptually comprehensive interface, in which aural and haptic clues play are used to supplement visual clues.

\section{References}

[1] Caliani, M., Pala, P., and Del Bimbo, A. (1998). Computer analysis of TV spots: The semiotics perspective. In Proceedings of the IEEE International Conference on Multimedia Computing and Systems, Austin, TX.

[2] Eco, U. (197). A Theory of Semiotics. Indiana University Press, Bloomington.

[3] Gombrich, E. H. (1965). Art and Mlusion. A study in the pshychology of pictorial representation. Pantheon Books.

[4] Gupta, A., Santini, S., and Jain, R. (1997). In search of information in visual media. Communications of the ACM, 40(12):35-42.

[5] Itten, J. (1961). The art of Color. Reinhold Pub. Corp., New York. 
[6] Kalisa, C. and Torrésani, B. (1993). N-dimensional affine Weyl-Heisenberg wavelets. Annales de L'Instut Henri Poincarè, Physique thèorique, 59(2):201-236.

[7] Lovelock, D. and Rund, H. (1975, 1989). Tensors, Differential Forms, and Variational Principles. Dover Books on Advanced Mathematics, 63. Dover Publications, Inc, New York.

[8] Ravi Kanth, K., Agrawal, D., and Singh, A. (1998). Dimensionality reduction for similarity searching in dynamic databases. In Proceedings of the 1998 ACM SIGMOD conference, pages 166-175.

[9] Riloff, E. and Hollaar, L. (1996). Text databases and information retrieval. ACM Computing Surveys, 28(1):133-135.

[10] Santini, S. (1998). Explorations in Image Databases. $\mathrm{PhD}$ thesis, Univerity of California, San Diego.

[11] Santini, S. and Jain, R. (1997). Similarity is a geometer. Multimedia Tools and Applications, 5(3). available at http://wwwcse.ucsd.edu/users/ssantini.

[12] Saussure, F. D. (1960). Cours de linguistique generale. Payot, Paris. 